\title{
Our microbiology coverage in 2020
}

\author{
Microbiology has been front and centre during the ongoing SARS-CoV-2 pandemic. We reflect on the content we \\ published this year and look ahead to aligning output with the Sustainable Development Goals in 2021.
}

n n our final Editorial of 2019, we promised to continue to curate and publish substantial advances across the breadth of fields covered in Nature Microbiology. We told our readers that we would need to become more selective, owing to increasing numbers of submissions, and that we would publish between 10-14 papers per issue. Here, we reflect on a year in the pages of Nature Microbiology, and set out our aspirations for 2021.

The year 2020 has been unforgettable. It is the year that the SARS-CoV-2 pandemic took hold. It is the year that microbiology became a topic of everyday conversation in school playgrounds, across fences between neighbours, on stairwells in apartment blocks, between parents, children and grandchildren, in the supermarket, on the news and on social media. Our submission volumes swelled from March onwards, by up to $25 \%$ month-for-month compared with 2019 , as researchers worked at breakneck pace to understand the biology of SARS-CoV-2. From pathogenesis, diagnostics, epidemiology, evolution, host responses and structural virology, to non-pharmaceutical interventions, public health policies, therapies and vaccines, we have had the challenging but rewarding task of reading hundreds of SARS-CoV-2 manuscripts and contributing to the communication of these findings as part of the global effort to tackle the pandemic.

Research into SARS-CoV-2 has undoubtedly skewed the diversity of papers we receive for consideration towards just one pathogen, but nevertheless we continue to strive to represent the astonishing breadth of topics encompassed within microbiology in our pages. Of the thousands of research papers submitted to us in 2020, we selected around $9-12 \%$ for external review. With the help of our peer reviewers, whom we thank unreservedly for their thorough scrutiny of manuscripts, advice and insights, we have published a total of 119 research articles online in 2020.
Altmetrics provides an aggregate measure of how our articles are viewed, used and discussed online. Eight of the top ten research manuscripts, as measured by Altmetrics, are unsurprisingly SARS-CoV-2 papers, with the most attention being paid to a paper reporting the evolutionary origins of the virus and finding that the viral lineage giving rise to SARS-CoV-2 has been circulating in bats for decades. We were also proud to publish the paper naming the virus as part of our continuing support for the importance of (viral) taxonomy.

As an illustration of the wider variety of papers we have published, we invite you to explore a small sample: how volatiles produced by Streptomyces attract soil arthropods that spread their spores; how fungi impact bacterial species in microbiomes; how a jumbo phage forms a nucleus-like structure to evade CRISPRCas DNA targeting; how a Staphylococcus aureus metabolite can reprogramme the host immune response during persistent infection; monkeypox emergence in chimpanzees; how you can apply two methods for single-cell RNA sequencing in bacteria; how host immune cells respond to Candida auris; steps towards engineering inducible nitrogenase activity into cereal endophytes to eventually improve crop yields; how an exported kinase remodels red blood cells in malaria; how to analyse the spatial metabolome of host-microbe interactions in situ (in a deep sea mussel) at the micrometre scale; how to determine antibiotic susceptibility and resistance of selected pathogens in just ten minutes; and how yeast collectively live at higher temperatures by cooperative secretion of glutathione.

Next year the ongoing pandemic will undoubtedly continue to feature in our pages, yet we remain committed to highlighting important advances in every area of our scope, both in our research papers and in selected and commissioned Comments, Perspectives and Reviews.
One area that we will endeavour to feature more prominently in our pages is research related to the 17 Sustainable Development Goals (SDGs) put forth by the United Nations. These two issues are not independent; the pandemic seems likely to exacerbate many global problems, not least because it is interfering with efforts to address them, as noted by the United Nations Development Programme. Before the pandemic, microbiologists have long been at the forefront of research to achieve many of the 17 SDGs (A. A. Akinsemolu et al. J. Clean. Prod. 182, 139-155; 2018), and going forward it will be crucial to renew efforts, work together and across multidisciplinary boundaries to achieve these goals. Editorially, we will endeavour to select for our readers outstanding primary research, accompanied by commissioned commentaries, that together align closely with SDGs to help to advance these efforts. Alongside this we are continuing our efforts to increase diversity among authors and reviewers, and hope that the benefits this brings will be reflected in our pages.

At the close of this year, we thank all of the researchers who have chosen to submit research or write commentaries, and all of our readers who have taken the time to read the work of their peers. This year we also thank three outgoing editors, Nonia Pariente, Paula Jauregui and Yassine Cherrak, for their contributions to our journal.

In 2021, please join us in reading the very best research across the entire scope of microbiology, much of which will undoubtedly continue to improve our knowledge and understanding of human, animal, plant, ocean and planetary health. Do get in touch to let us know how you think we're doing, both in terms of covering the entire field and whether we should focus on SDGs, and if so, what types of research are best poised to contribute to those goals.

Published online: 19 November 2020 https://doi.org/10.1038/s41564-020-00828-1 